\title{
Strongly Dispersive Transient Bragg Grating for High Harmonics
}

\author{
J. P. Farrell, ${ }^{1,2}$ L. S. Spector, ${ }^{1,2}$ M. B. Gaarde, ${ }^{1,3}$ B. K. \\ McFarland ${ }^{1,2}$, P. H. Bucksbaum, ${ }^{1,2}$ and Markus Gühr ${ }^{1,2 *}$ \\ ${ }^{1}$ Stanford PULSE Institute, SLAC National Accelerator Laboratory, Menlo Park, CA 94025, USA \\ ${ }^{2}$ Departments of Physics and Applied Physics, \\ Stanford University, Stanford, CA 94305, USA \\ ${ }^{3}$ Department of Physics and Astronomy, \\ Louisiana State University, Baton Rouge, LA 70803-4001 USA \\ *Corresponding author: mguehr@stanford.edu
}

(Dated: May 5, 2010)

We create a transient Bragg grating in a high harmonic generation (HHG) medium using two counterpropagating pulses. The Bragg grating disperses the harmonics in angle and can diffract a large bandwidth with temporal resolution limited only by the source size.

Production of high harmonics in a strong laser field is caused by atomic ionization followed by laser-driven propagation and coherent electron-ion recombination [1-3]. In high harmonic spectroscopy (HHS), one uses the harmonic amplitude and phase to gain information about the electronic quantum state of the source molecules or atoms [4-6]. For example, HHS has been utilized to demonstrate multiple orbital contributions to the harmonics [7-9]. The HHS technique could be extended in principle to transient processes on photoexcited electronic states that play a crucial role in photochemistry. However, such processes are weak-field phenomena, which limits the overall excited state population and the HHS sensitivity to the excitation.

Transient gratings are generally applied in the IR to UV range to increase the sensitivity of measurements to the excited atomic/molecular states. Two excitation pulses, intersecting under an angle $\alpha$, create an excitation grating in the sample while a third (probe) pulse is diffracted from the grating. The diffracted signal has high sensitivity to excited state dynamics $[10,11]$. This technique has been successfully transferred to the high harmonic domain $[12,13]$, using a very small angle $\alpha$ between the intersecting grating pulses. This resulted in a spatially modulated HHG signal from the probe pulse. The sidebands showed increased 
excited state sensitivity and consisted of several harmonics, all spatially overlapped.

In this paper we present a different transient grating scheme in which the harmonics are not only deflected into a sideband, but are also dispersed in angle within a diffraction order, to enable HHS analysis. This is achieved by enlarging the angle $\alpha$ to $180^{\circ}$ resulting in a shorter grating period $d$, which disperses the harmonics to distinguishable angles without an additional grating element. We have observed up to seven distinguishable harmonics at their respective Bragg angles of the laser-induced transient grating. The diffracted harmonics range from the 19th to the 31st of the fundamental $800 \mathrm{~nm}$ light, which corresponds to a $45 \%$ energy bandwidth.

Figure $1 \mathrm{~A}$ shows the counterpropagating grating beams $\mathbf{k}_{\mathbf{1}}$ and $\mathbf{k}_{\mathbf{2}}\left(k_{1,2}=2 \pi / \lambda\right)$ that create a standing wave with a period $d=\lambda / 2$ and reciprocal lattice vector $k_{G}=2 \pi / d$. A probe pulse with wavelength $\lambda_{p}$ and wave vector $\mathbf{k}_{\mathbf{p}}$ is focused in the grating and harmonics of order $\mathrm{n}$ with wavelength $\lambda_{H H}=\lambda_{p} / n$ are produced. Since we are operating in the thick grating or Bragg regime [14], a strong diffraction of the harmonics is only observed at their Bragg angle $\theta_{B}(1 \mathrm{~A})$. For $\lambda_{p}=\lambda$, i.e. grating and probe beam of equal color as implemented in our experiment, the phase matching diagram in Fig. 1A indicates a Bragg peak for harmonic $\mathrm{n}$ under the angle:

$$
\sin \theta_{B}=\frac{k_{G}}{2 k_{H H}}=\frac{\lambda_{H H}}{\lambda_{p}}=\frac{1}{n}
$$

The probe beam is focused into the interaction region so $\mathbf{k}_{\mathbf{p}}$ is distributed over a range of angles, allowing Bragg diffraction of several harmonics at different angles. In order to resolve diffracted harmonics, the divergence of a single harmonic Bragg peak must be smaller than the separation of neighboring harmonic Bragg peaks.

Figure 1B shows the experimental setup. We divide $230 \mu \mathrm{J}$ pulses from an amplified Ti:Sapph laser system (30 fs, $800 \mathrm{~nm}, 1 \mathrm{kHz}$ rep. rate) into two grating beams and a probe beam in the ratio 1:1:2. The pulses are focused onto a gas jet inside a vacuum chamber with different confocal parameters resulting in intensities of $1 \times 10^{14} \mathrm{~W} / \mathrm{cm}^{2}$ and $3 \times 10^{14} \mathrm{~W} / \mathrm{cm}^{2}$ for the grating and probe beams respectively. The spatial and temporal overlap of all three pulses is indicated by enhanced plasma generation in Ar gas. A movable detector arm with Al filters for rejecting the fundamental probe wavelength is equipped with a cooled and VUV sensitive CCD camera. The arm can be adjusted either to the angle of the unscattered probe and its harmonics (zero order) or to the Bragg diffracted signal. 
When the three pulses are overlapped in time, we can optimize the diffraction of harmonic 21 by adjusting the incident angle of the probe pulse to $1 / 21=48 \mathrm{mrad}$. A maximum diffraction efficiency above 10\% is measured by comparing the intensities of the zero order and the Bragg diffracted signal. Figure 2A shows the scattered Bragg peaks as a function of angle with respect to the grating normal. Seven harmonics, corresponding to a $45 \%$ bandwidth are distinguishable. Towards higher harmonics the modulation contrast vanishes. Superimposed on the peaks are the calculated Bragg angles for the harmonics using Eq 0.1. This indicates that the peaks can be interpreted as dispersed harmonics. The single adjustable parameter in this comparison is the zero on the horizontal scale of Fig. 2, which cannot be measured with sufficiently high accuracy in the experiment. We shifted the zero angle for best coincidence with the diffraction angles predicted by Eq. 0.1. The relative angle is known accurately because it corresponds to the ratio of the relative displacement of the CCD pixel to the distance of the detector - interaction region.

To show that the distinct peaks correspond to dispersed harmonics of different wavelengths, we performed a coherent diffraction experiment by translating a $50 \mu \mathrm{m}$ slit through the angular peaks in Fig. 2B and collecting the diffraction images on the same CCD camera. The widths of the central diffraction peaks, given as the circles in Fig. 2B, decrease as we move the slit to smaller angles. This shows that the wavelength of the peaks becomes shorter at smaller angles. A comparison of the measured diffraction width with the predicted Fraunhofer width for each harmonic (solid square in Fig. 2B) shows good agreement, which strongly supports our interpretation that the peaks are different dispersed harmonics. The systematic differences between experiment and theory for the edges of the spectral region result from the relatively broad linewidths of the harmonics and a background from the scattered zero order.

Figure 3A illustrates the light induced grating mechanism, showing the intensity distribution in the focal region of the grating beams when overlapped with the probe beam. The two counterpropagating grating beams form a standing wave. When the probe beam is added, it is only along the nodes of this grating ( $\mathrm{x}= \pm 0.2, \pm 0.6 \mu \mathrm{m}$ in Fig. $3 \mathrm{~A})$ that a forward-traveling wave exists with constant intensity along the propagation direction. At the antinodes, the field superposition results in an egg crate-like intensity pattern with half wavelength periodicity ( $\mathrm{x}=0, \pm 0.4, \pm 0.8 \mu \mathrm{m}$ in Fig. $3 \mathrm{~A}$ ). Efficient high harmonic phase matching is only achieved in regions of the grating where the intensity modulation along the propagation 
direction is small $[15,16]$, which means that no harmonics can build up along the grating antinodes.

We have calculated the macroscopic harmonic signal from an argon gas jet in a transient grating configuration similar to that of Fig. 3A, by periodically lowering the argon density in the anti-nodes of the grating. We solve the coupled wave equation and time-dependent Schrödinger equation, within the strong field approximation $[17,18]$, with one transverse dimension to allow the description of the probe beam incident at a non-zero angle. With the reduced dimensionality a Gaussian beam focuses more slowly. Thus, for best comparison with the experimental setup, we choose a slightly smaller focal diameter $(28 \mu \mathrm{m})$ and slightly larger confocal parameter $(2.6 \mathrm{~mm})$. To achieve the same range of harmonics in the Bragg peak, which is determined by the beam divergence, we choose a smaller incidence angle of $37 \mathrm{mrad}$. Figure 3B shows the far field spectral-angular distribution of harmonics generated by a $30 \mathrm{fs}, 2.3 \times 10^{14} \mathrm{~W} / \mathrm{cm}^{2}$ pulse. Figure $3 \mathrm{C}$ shows the spectrally integrated angular distribution, which offers a more direct comparison to and shows good qualitative agreement with the experimental result in Fig. 2A . It is clear from the calculation that the harmonics are well resolved in angle and that the different peaks in the angular distribution can be assigned to different harmonic orders. We find that the angular separation is directly related to the spectral separation of the harmonics, so that shorter pulses or higher intensities can lead to more angular blurring [19]. The angular distribution in the calculation is slightly offset from the Bragg prediction (circles in B) and the offset increases with order. We attribute this to a dipole phase matching effect which is likely exaggerated in one transverse dimension. We will explore this further in a forthcoming publication.

Our results indicate that we have both temporal and spectral resolution from the Bragg grating. The diffracted bandwidth is wide enough to identify the relatively sharp structural minima from molecular electronic wavefunctions in HHS [20]. Apart from the use in HHS, the high efficiency will also allow the scheme to be useful for switching the angle of an HHG output within a short time interval. We thank J. P. Marangos and K. J. Schafer for helpful and stimulating discussions. We thank the Department of Energy, Office of Basic Energy Science for support through the Stanford PULSE Institute and the National Science Foundation for support through grant PHY-0449235. L. S. S. acknowledges the support of 
a NDSEG fellowship.

[1] P. B. Corkum. Plasma perspective on strong field multiphoton ionization. Phys. Rev. Lett., 71:1994-1997, 1993.

[2] K. J. Schafer, Baorui Yang, L. F. DiMauro, and K. C. Kulander. Above threshold ionization beyond the high harmonic cutoff. Phys. Rev. Lett., 70(11):1599-1602, Mar 1993.

[3] K. C. Kulander, K. J. Schafer, and J. L. Krause. Theoretical model for intense field high-order harmonic generation in rare gases. Laser Physics, 3:359 - 64, 1993.

[4] M. Lein, N. Hay, R. Velotta, J. P. Marangos, and P. L. Knight. Role of the intramolecular phase in high-harmonic generation. Physical Review Letters, 88(18):183903 - 4, MAY 2002.

[5] A. T. Le, R. R. Lucchese, and C. D. Lin. Uncovering multiple orbitals influence in high harmonic generation from aligned $\mathrm{N}_{2}$. J. Phys. B, 42:211001, 2009.

[6] J. Itatani, J. Levesque, D. Zeidler, H. Niikura, H. Pepin, J. C. Kieffer, P. B. Corkum, and D. M. Villeneuve. Tomographic imaging of molecular orbitals. Nature, 432(7019):867 - 871, Dec 2004.

[7] B.K. McFarland, J.P. Farrell, P.H. Bucksbaum, and Gühr M. High Harmonic Generation from Multiple Orbitals in $\mathrm{N}_{2}$. Science, 322(5905):1232, 2008.

[8] W. Li, X. Zhou, R. Lock, S. Patchkovskii, A. Stolow, H.C. Kapteyn, and M.M. Murnane. Timeresolved dynamics in $\mathrm{N}_{2} \mathrm{O}_{4}$ probed using high harmonic generation. Science, 322(5905):1207, 2008 .

[9] O. Smirnova, Y. Mairesse, S. Patchkovskii, N. Dudovich, D. Villeneuve, P. Corkum, and M.Y. Ivanov. High harmonic interferometry of multi-electron dynamics in molecules. Nature, 460(7258):972-977, 2009.

[10] MD Fayer. Dynamics of molecules in condensed phases: picosecond holographic grating experiments. Annual Review of Physical Chemistry, 33(1):63-87, 1982.

[11] S. Mukamel. Principles of nonlinear optical spectroscopy. Oxford University Press New York, 1995.

[12] Y. Mairesse, N. Dudovich, J. Levesque, D. Kartashov, D. M. Villeneuve, P. B. Corkum, and T. Auguste. Transient phase masks in high-harmonic generation. Optics Letters, 32(4):436 438, Feb 2007. 
[13] Y. Mairesse, D. Zeidler, N. Dudovich, M. Spanner, J. Levesque, D. M. Villeneuve, and PB Corkum. High-Order Harmonic Transient Grating Spectroscopy in a Molecular Jet. Physical Review Letters, 100(14):143903, 2008.

[14] HJ Eichler, P. Gunter, and DW Pohl. Laser-induced dynamic gratings. Springer, 1987.

[15] P. Balcou, P. Salieres, A. LHuillier, and M. Lewenstein. Generalized phase-matching conditions for high harmonics: the role of field-gradient forces. Physical Review A (Atomic, Molecular, and Optical Physics), 55(4):3204 - 10, APR 1997.

[16] X. Zhang, A.L. Lytle, T. Popmintchev, X. Zhou, H.C. Kapteyn, M.M. Murnane, and O. Cohen. Quasi-phase-matching and quantum-path control of high-harmonic generation using counterpropagating light. Nature Physics, 3(4):270-275, 2007.

[17] M. Lewenstein, Ph. Balcou, M. Yu. Ivanov, Anne L'Huillier, and P. B. Corkum. Theory of high-harmonic generation by low-frequency laser fields. Phys. Rev. A, 49(3):2117-2132, Mar 1994.

[18] M.B. Gaarde, J.L. Tate, and K.J. Schafer. TOPICAL REVIEW: Macroscopic aspects of attosecond pulse generation. Journal of Physics B Atomic Molecular Physics, 41(13):2001, 2008 .

[19] At intensities up to $3 \times 10^{14} \mathrm{~W} / \mathrm{cm}^{2}$ the harmonics are still angularly resolved, but the curve in Fig. 3C becomes strongly dominated by H21 and H23. This is because of the particular shape of the spectrum in our calculation, partly caused by suppressing the long trajectory contribution (since it is in general overestimated by the strong field approximation).

[20] C. Vozzi et al.. Controlling Two-Center Interference in Molecular High Harmonic Generation. Physical Review Letters, 95:153902, 2005. 

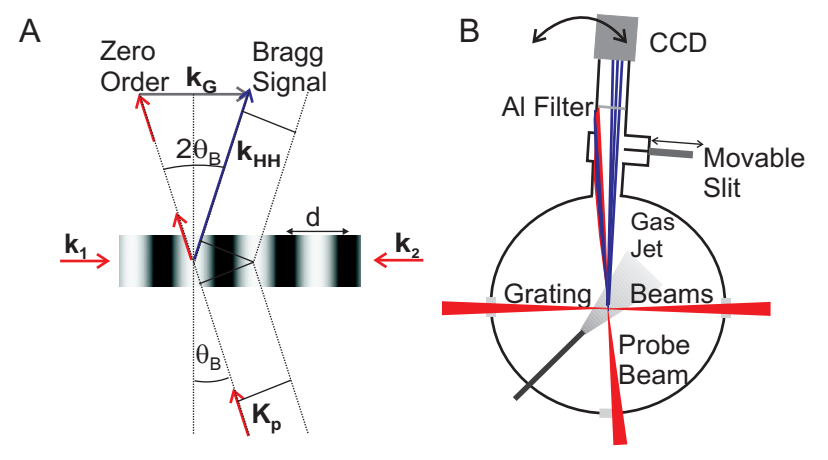

FIG. 1: Experimental diagram showing the geometry of the Bragg scheme (A) and the apparatus (B).

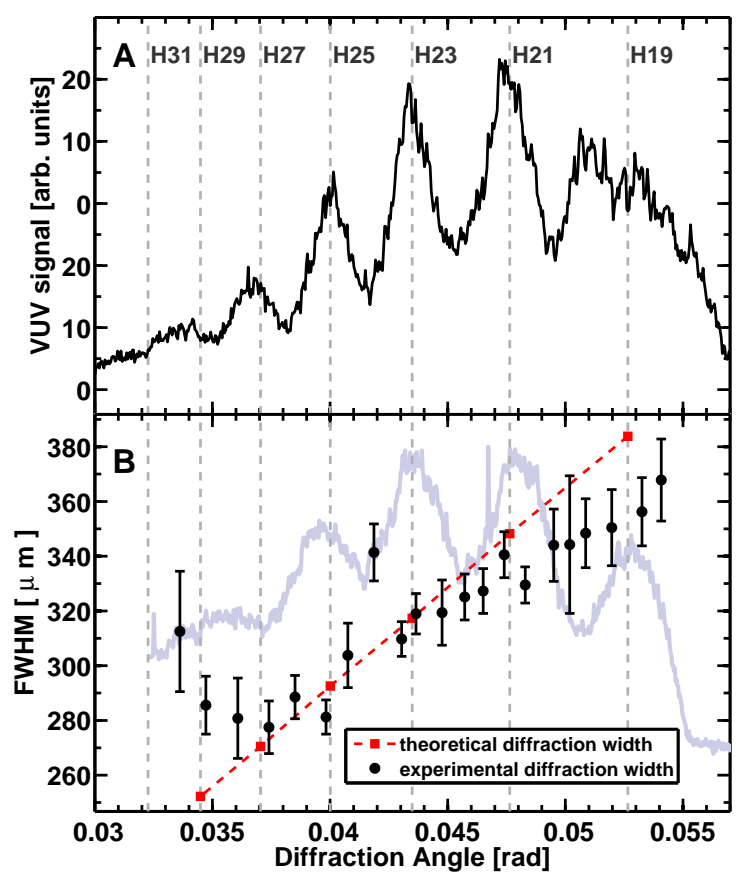

FIG. 2: (A) Bragg order showing up to seven distinct peaks, which we interpret as distinct harmonics. (B) Widths of the slit diffraction pattern (black circles) of a different spectrum than shown in A (blue) and predictions based on harmonic wavelength (red squares). 

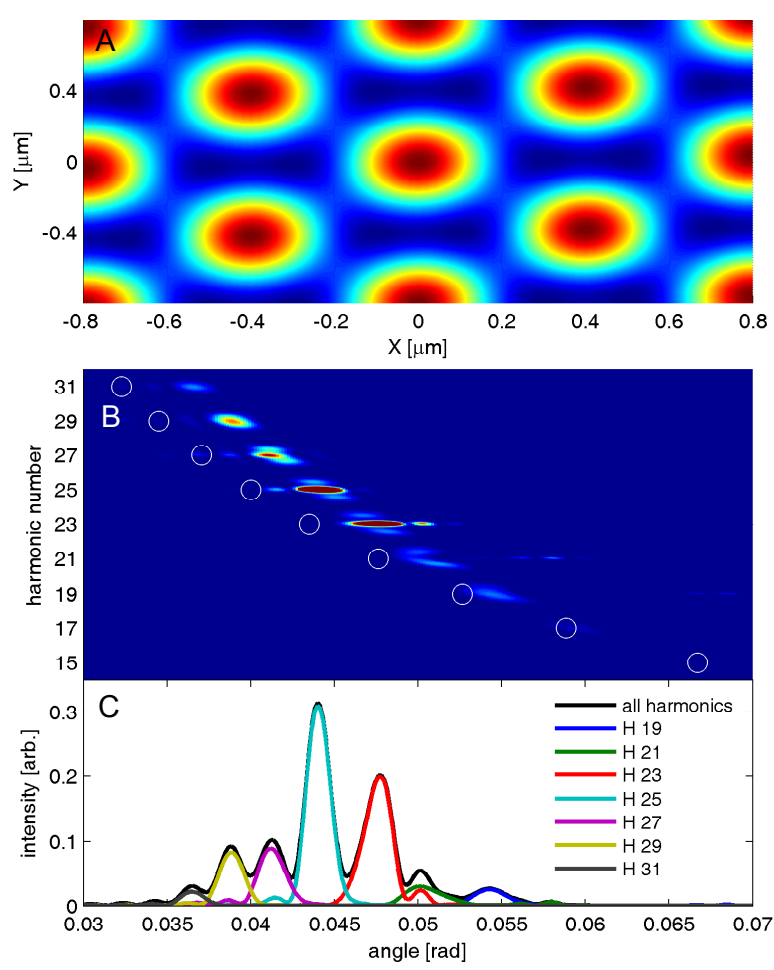

FIG. 3: (A)Intensity distribution of the three pulse overlap. The grating beams propagate along $\pm x$, and the probe beam hits the $y$-axis under the Bragg angle for harmonic 21.(B) Macroscopic calculation of the far field harmonic yield vs angle and harmonic order, shown on a linear color scale. The circles indicate the angles predicted by the Bragg law. (C) Spectrally integrated angular distribution for all harmonics above the 19th (black line), to be compared directly with Fig. 2A. The yield vs angle for individual harmonics is also shown (each integrated over twice the fundamental frequency, different colors). 